\title{
Studying Diquark Correlations in the Nucleon by Means of Lattice QCD Simulations
}

\author{
P. Faccioli*, M. Cristoforetti, M. Traini \\ Physics Department and I.N.F.N. Gruppo Collegato Trento, Facoltá di Scienze, Universitá degli \\ Studi di Trento, Via Sommarive 15, I-38050 Povo (Trento), Italy. \\ European Centre for Theoretical Studies in Nuclear Physics and Related Areas, Via Sommarive \\ 284, I-38050 Villazzano (Trento), Italy
}

\section{G. Ripka}

Service de Physique Theorique, Centre d'Etudes de Saclay, F-91191, Giffur-Yvette Cedex, France.

faccioli@science.unitn.it, cristofescience.unitn.it,

traini@science.unitn.it, ripka@cea.fr

In this presentation we review our recent attempt to address two questions concerning dynamical correlations inside the nucleon: (i) is the non-perturbative quark-quark interaction particularly attractive in the color antitriplet $0^{+}$diquark channel? (ii) are such correlations induced by instantons? We define appropriate ratios of Euclidean three-point functions which encode specific information about two-body correlations and can be computed on the lattice. We analyze such correlations functions in different models with very different diquark content: a naive non-relativistic SU(6) quark model, the Random Instanton Liquid Model and the Quark Chiral Soliton Model. We show that such models lead to radically different predictions. We conclude that a lattice QCD calculation of such correlation functions can determine which picture is most realistic.

XXIIIrd International Symposium on Lattice Field Theory

25-30 July 2005

Trinity College, Dublin, Ireland

\footnotetext{
*Speaker.
} 

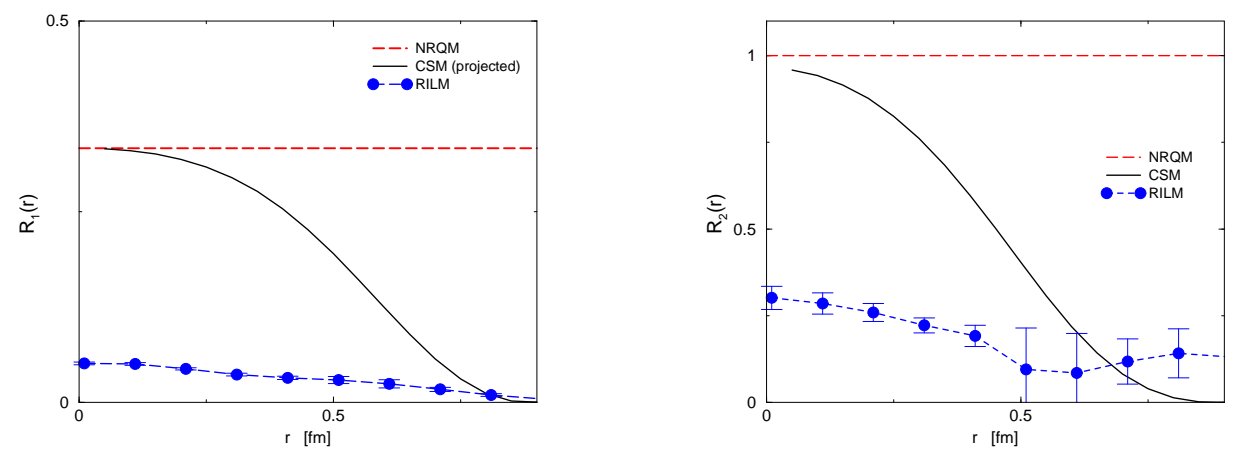

Figure 1: Predictions for the ratios $R_{1}(r)=\rho_{\gamma_{3}}(\mathbf{r}) / \rho_{\gamma_{5}}(\mathbf{r})$ (LEFT PANEL) and for the ratio $R_{2}(r)=$ $\rho_{\gamma_{5} \gamma_{5}}(\mathbf{r}) / \rho_{\gamma_{5}}(\mathbf{r})$ (RIGHT PANEL) obtained in the Non-Relativistic Quark Model (NRQM), in the Random Instanton Liquid Model (RILM) and in the Chiral Soliton Model (CSM).

\section{Introduction}

The question if the non-perturbative interaction in QCD is characterized by a particularly strong attraction in the color anti-triplet $0+$ diquark channel has long been debated. From a phenomenological perspective, some evidence in this direction has been gathered in the last decades (see [1] for a recent review). For example, it has been observed that the anti-triplet $0^{+}$diquarks play an important role in non-leptonic weak decays of both hyperons and kaons [2, 3]. From a more fundamental perspective, however, a conclusive proof is still missing. Lattice gauge theory represents the only presently available tool for performing non-perturbative calculations in QCD and can be used to investigate the role of diquark correlations, from first principles.

In this talk we review a recent investigation on diquarks correlations in the nucleon[4]. For alternative studies on diquark correlations in vacuum or in heavy-light hadrons see the contributions of Orginos and Lucini, in this conference. We identify and study a set of lattice-calculable matrix-elements, which are very sensitive to the spin-flavor structure of the quark-quark interaction. In order to show that these matrix elements encode important information on diquark correlations inside hadrons, we compute them using three different phenomenological models: a naive Non-Relativistic Quark Model, in which dynamical diquark correlations are absent, the Random Instanton Liquid Model, where $0^{+}$diquarks are strongly favored by the 't Hooft interaction, and a Chiral Soliton Model, where the 't Hooft interaction is treated at the mean-field level, hence with no dynamical two-body correlations. We show that these models lead to very different predictions.

In addition, we also propose a lattice calculation to check if the strong attraction in the $0^{+}$ anti-triplet diquark channel is mediated by instantons.

\section{Diquark density matrix elements}

Direct insight on quark-quark correlations inside specific hadrons can be gained by focusing on two-body local density operators, which simultaneously probe the position and the discrete quantum numbers of two quarks. In this view, we consider a set of four-field operators (which we shall refer to as diquark densities), in which two quarks with quantum numbers of a $0^{+}$and $1^{+}$ color anti-triplet diquark are destroyed and re-emitted, in the same point. 
We work with Euclidean space-time and we define $D_{\Gamma}(\mathbf{r})=F_{\Gamma}^{a \dagger}(\mathbf{r}, 0) F_{\Gamma}^{a}(\mathbf{r}, 0)$, with $F_{\Gamma}^{a}(x)=$ $\varepsilon^{a b c} u_{a}(x) C \Gamma d_{b}(x) . C=i \gamma_{2} \gamma_{4}$ is the charge-conjugation operator, $\Gamma \in\left\{1, \gamma_{5}, \gamma_{\mu}, \gamma_{\mu} \gamma_{5}, \sigma_{\mu, v}\right\}$ and $a, b, c$ are color indices. The operator $F_{\Gamma}^{a}(x)$ absorbs two quarks in a point with quantum numbers of a color anti-triplet diquark. For example, $F_{\gamma_{3}}^{a}(x)$ annihilates a $1^{+}$diquark, while $F_{\gamma_{5}}^{a}(x)$ absorbs $0^{+}$diquarks. Hence, the matrix element:

$$
\rho_{\Gamma}(\mathbf{r})=\left\langle P\left|D_{\Gamma}(\mathbf{r})\right| P\right\rangle
$$

measures the probability amplitude to find at time $t=0$ two quarks at the point $\mathbf{r}$ in the proton, in a anti-triplet color state and with quantum numbers specified by $\Gamma$.

The choice of $\Gamma$ determines the transformation property of the $F_{\Gamma}^{a}(x)$ operator under exchanges of the flavor indices. For $\Gamma=\gamma_{5}$, the diquark state is an iso-scalar state. When $\Gamma=\gamma^{4}$ the diquark state is an iso-vector state.

In the following section we discuss how the comparison of the different matrix elements (2.1) can provide useful insight about the strength and the spin- and flavor- structure of the quark-quark interaction.

\subsection{Are the correlations $0+, \overline{3}$ channel particularly strong?}

If the quark-quark interaction is particularly attractive in the $0^{+}$anti-triplet diquark channel we expect that the $0^{+}$diquark density $\rho_{\gamma_{5}}(\mathbf{r})$ should be enhanced with respect to a scenario in which the interaction is not particularly attractive. In fact, the $u$ and $d$ quarks would have a larger probability "to be found" in the same point and be destroyed by the local operator $F_{\gamma_{5}}^{a}(x)$.

Hence, in a scenario in which $0^{+}$diquark correlation are particularly strong we expect that the ratio

$$
R_{1}(\mathbf{r})=\frac{D_{\gamma_{4}}(\mathbf{r})}{D_{\gamma_{5}}(\mathbf{r})}
$$

should be suppressed with respect to a scenario in which direct dynamical correlations are not particularly strong in this channel.

To quantify this statement let us compare the predictions for this ratio obtained in the three models discussed above. The results of our phenomenological calculations are shown in Fig. 1(left panel) ${ }^{1}$. In the $S U(6)$ Non-Relativistic Quark Model, in which there are not dynamical diquark correlations, this ratio is completely determined by the $S U(6)$ spin-flavor structure of the wavefunction, and is identically equal to $1 / 3$. In the Random Instanton Liquid Model, $R_{1}(\mathbf{r})$ is sizebly reduced in magnitude (by a factor $\simeq 5$ ). The prediction of the Chiral Soliton Model is very different from that of the Random Instanton Liquid Model Inside the soliton (i.e. for $|\mathbf{r}| \lesssim 0.7-0.8 \mathrm{fm}$ ). In particular, at $\mathbf{r}=0$ the $p$-wave contribution from the lower components of the spinors vanishes and one recovers the Non-Relativistic Quark Model Results. The $1^{+}$diquark density in the soliton drops down very rapidly at the border, where the pion field is most intense.

These results can be interpreted as follows. In the Random Instanton Liquid Model, the spinand flavor- dependent 't Hooft interaction generates a strong attraction which enhances the probability amplitude of finding two quarks in the same point in the $0^{+}$anti-triplet configuration, relative

\footnotetext{
${ }^{1}$ We refer the interested reader to our original work for all the details of the calculations
} 
to the amplitude of finding them in the $1^{+}$configuration. This explains why the Random Instanton Liquid Model prediction for $R_{1}(\mathbf{r})$ is much smaller than that of the $S U(6)$ Non-Relativistic Quark Model and the Chiral Soliton Model.

Based on this discussion we conclude that, if the non-perturbative QCD interactions generate a strong correlation in the $0^{+}$anti-triplet channel, then we predict that the curve obtained from a lattice calculation should lie much below $1 / 3$. If lattice simulations found that $R_{1}(\mathbf{r}) \sim 1 / 3$, than this would imply that diquarks are not particularly correlated in the $0^{+}$diquark channel. $R_{1}(\mathbf{r})>1 / 3$ would represent an indication that the quark-quark interaction is less attractive in the $0^{+}$channel, relative to the $1^{+}$channel. This would certainly be a very surprising result, since the $\left(\overline{\mathbf{3}}_{c}, \overline{\mathbf{3}}_{f}\right)$ channel is favored by both the perturbative and the instanton-mediated interactions.

\subsection{Are the diquark $0+, \overline{3}$ correlations induced by instantons?}

The ratio $R_{1}(\mathbf{r})$ does not encode information about the microscopic dynamical mechanism underlying such diquark correlations. In fact, two completely different quark-quark effective interactions (e.g. one with a chirality-conserving vertex and one with a chirality-flipping vertex) may lead to the same predictions, as long as the short-range attraction in the $0^{+}$channel is sufficiently strong.

In order to gain some insight on the microscopic origin of diquarks we need to analyze a different ratio:

$$
R_{2}(\mathbf{r})=\frac{\rho_{\gamma_{5} \gamma_{4}}(\mathbf{r})}{\rho_{\gamma_{5}}(\mathbf{r})}
$$

The results of our calculations in the three phenomenological models are reported in Fig. 1 (RIGHT PANEL). In the Non-Relativistic Quark Model, both the $\rho_{\gamma_{5}}(\mathbf{r})$ and $\rho_{\gamma_{4} \gamma_{5}}(\mathbf{r})$ densities probe the $0^{+}$diquark content of the proton, so $R_{2}(\mathbf{r})=1$. Also the Random Instanton Liquid Model prediction remains almost constant, but its magnitude is smaller than the Non-Relativistic Quark Model, by a factor 3 or so. The fact that, in the Random Instanton Liquid Model, $R_{2}(\mathbf{r}) \ll 1$ has an important dynamical explanation. It is due to the different sensitivity of the numerator and denominator to the so-called direct-instanton contribution. The $\rho_{\gamma_{5}}(\mathbf{r})$ diquark density receives maximal contribution from the interaction of quarks with the field of the closest (direct) instanton, in the vacuum. This statement can be verified by computing the correlator in the single-instanton approximation, discussed in [6]. On the other hand, the density in the denominator, $\rho_{\gamma_{5} \gamma_{4}}(\mathbf{r})$ does not receive such a direct-instanton contribution and instanton-induced effects come only from the interactions of quarks with many instantons. The magnitude of the latter contributions are parametrically suppressed by the diluteness of the instanton vacuum.

We remark that the very strong channel dependence of hadronic correlation functions is a well-known dynamical implication of instanton models. It is quite hard to obtain this effect in alternative dynamical mechanisms. In the Chiral Soliton Model, the ratio $R_{2}(\mathbf{r})$ remains of order 1 for $|\mathbf{r}| \lesssim 1 \mathrm{fm}$ and drops rapidly at the border of the soliton. The significant deviation of the Chiral Soliton Model result from the Random Instanton Liquid Model prediction shows that a mean-field approach does not capture correlations associated to the direct-instanton effects.

From this discussion it follows that, if the scalar diquark correlations are mainly induced by instantons or in general by a Nambu-Jona-Lasinio type of interaction (i.e. chirally symmetric, with a chirality flipping vertex), then a lattice measurement should give $\rho_{\gamma_{4} \gamma_{5}}(\mathbf{r}) \ll \rho_{\gamma_{5}}(\mathbf{r})$, so $R_{2}(\mathbf{r}) \ll 1$. 


\section{Diquark densities on the lattice}

In this section we discuss how the diquark densities defined above can be computed in lattice QCD. It should be mentioned that this approach does not only apply to Lattice calculations. In fact, the same approach was used to compute the diquark densities matrix elements in the Random Instanton Liquid Model, in which the Monte Carlo average over all gauge configurations is replaced by and average over the configurations of an instanton liquid ensemble [5]. We start by considering the following Euclidean three-point correlation function:

$$
G^{\Gamma}\left(x_{i}, x_{f}, y\right)=\left\langle 0\left|J^{\alpha}\left(x_{f}\right) D_{\Gamma}(y) \bar{J}^{\alpha}\left(x_{i}\right)\right| 0\right\rangle,
$$

where $D_{\Gamma}(y)$ is the diquark density operator, and $J^{\alpha}(x)=\varepsilon_{a b c} u_{a}^{T}(x) C \gamma_{5} d_{b}(x) u_{c}^{\alpha}(x)$ is the usual interpolating operator which excites states with the quantum numbers of the proton.

The correlator (3.1) represents the probability amplitude to create a state with the quantum numbers of a proton at point $x_{i}$, to absorb and re-emit two quarks in a given diquark configuration at a the point $y$, and to finally re-absorb the three-quark state at the point $x_{f}$.

Next, we insert two complete sets of eigenstates of the QCD Hamiltonian in (3.1) and parametrize the matrix elements of diquark densities as:

$$
\left\langle N\left(\mathbf{p}^{\prime}, s\right)\left|D_{\Gamma}(y)\right| N(\mathbf{p}, s)\right\rangle=h_{\Gamma}\left(q^{2}\right) e^{-i q \cdot y} \bar{v}_{s^{\prime}}\left(\mathbf{p}^{\prime}\right) v_{s}(\mathbf{p}) .
$$

We obtain, after some straightforward manipulation [4]:

$$
G^{\Gamma}\left(x_{i}, x_{f}, y\right)=\Lambda^{2} \int d^{4} z R_{\Gamma}(y-z) \operatorname{Tr}\left[S\left(x_{f} ; z\right) S\left(z ; x_{i}\right)\right],
$$

where $S\left(x^{\prime}, x\right)$ is the nucleon propagator in coordinate space and $R(y)$ is the 4-dimensional Fourier transform of $h_{\Gamma}\left(q^{2}\right)$.

The physical interpretation of this result is the following. In the large Euclidean separation limit, the correlator $G^{\Gamma}\left(x_{i}, x_{f}, y\right)$ is parametrized by the function $R_{\Gamma}(x)$, which encode information about the probability amplitude to find the diquark at a given distance from the center of the nucleon. The convolution of $R_{\Gamma}(x)$ with the trace of proton propagators takes into account the de-localization of the position of the center of mass of the proton.

In order to clarify the relationship between the correlator (3.1) and the diquark density (2.1) it is instructive to consider first the static approximation for the nucleon, in which $M \rightarrow \infty$. In this limit Eq. (3.3) becomes $G^{\Gamma}\left(x_{i}, x_{f}, y\right)=2 \Lambda^{2} e^{-\left(x_{f}^{4}-x_{i}^{4}\right) M} \delta\left(\mathbf{x}_{\mathbf{f}}-\mathbf{x}_{\mathbf{i}}\right) \int d z_{4} R_{\Gamma}\left(\mathbf{r}, z_{4}\right)$. where we have used translational invariance to set $y_{4}=0$ and we have introduced $\mathbf{r}:=\mathbf{y}-\mathbf{x}_{\mathbf{i}}$, the distance between the center of the proton and the position where the diquark is absorbed. The last integral term in this expression represents the time-integrated probability amplitude to find a diquark at a distance $\mathbf{r}$ from the center of the nucleon. We can therefore identify this quantity with the diquark density (2.1) defined in section (2):

$$
\rho_{\Gamma}(\mathbf{r})=\int d z_{4} R_{\Gamma}\left(\mathbf{r}, z_{4}\right) .
$$

Hence, for an infinitely heavy nucleon, the expression relating the correlation function (3.1) to the diquark density is simply: $G^{\Gamma}\left(x_{i}, x_{f}, y\right)=2 \Lambda^{2} e^{-\left(x_{f}^{4}-x_{i}^{4}\right) M} \delta\left(\mathbf{x}_{\mathbf{f}}-\mathbf{x}_{\mathbf{i}}\right) \rho_{\Gamma}(\mathbf{r})$. 
If the nucleon mass is kept finite, there are corrections to the static limit expression (3.4), arising from replacing the static propagator with the exact expression. As a result, the convolution function $\operatorname{Tr}\left[S\left(x_{f} ; z\right) S\left(z, x_{i}\right)\right]$ in (3.3) - which determines the position of the center of mass of the nucleon - is de-localized on a volume which depends on the Euclidean time $\tau$. It is straightforward to verify that for a typical mass $M \simeq 1 \mathrm{GeV}$ and a typical Euclidean separation $\tau \simeq 1 \mathrm{fm}$ the position of the center of mass of the nucleon is smeared on a volume of radius $\simeq 0.3 \mathrm{fm}$, centered around the origin, therefore rather small. From this discussion we conclude that ratios of diquark densities can be estimated with an accuracy of $\delta \mathbf{r} \sim 0.3 \mathrm{fm}$ by computing ratios of correlation functions $G^{\Gamma^{\prime}}\left(x_{f}, x_{i}, y\right) / G^{\Gamma}\left(x_{f}, x_{i}, y\right)$ with Euclidean time separations between nucleon overlap operators and diquark density operators of the order of the fm. For example, in the Random Instanton Model calculations shown in the previous session, we have computed (3.1) choosing $\mathbf{x}_{i}=\mathbf{x}_{\mathbf{f}}=0, y=$ $(0,0, r, 0)$ and $x_{f}^{4}=-x_{i}^{4}:=\tau$, with $\tau=1 \mathrm{fm}$.

Notice that taking ratios allows to maximally reduce the effects of the corrections due to center of mass de-localization. In fact, such corrections affect in the same way both the numerator and the denominator and do not change the normalization of the correlation function.

\section{4. conclusions}

In this work we have addressed the question of how is it possible to study two-body diquark correlations in hadrons, using lattice QCD. We have identified some suitable lattice-calculable correlation functions, which allow to probe directly the diquark content of the nucleon. In particular, the ratio $R_{1}(\mathbf{r})$, defined in Eq. (2.2), measures the strength of the correlations in the scalar diquark channel, relative to that in the $1^{+}$channel. We predict that a lattice measurement must lead to $R_{1}(\mathbf{r}) \ll 1 / 3$ if scalar diquark correlations are dynamically enhanced.

The ratio $R_{2}(\mathbf{r})$, defined in Eq. (2.3), can be used to check the hypothesis according to which diquark correlations are mediated by instantons. We have argued that, in this case, we expect that lattice measurements should give $R_{2}(\mathbf{r}) \ll 1$.

We have computed these ratios using three phenomenological models. We have found that they lead to radically different predictions for the matrix elements we have selected. Hence, a lattice measurement could point out which picture is most realistic.

\section{References}

[1] R. Jaffe and F. Wilczek, Quarks, Diquarks and Pentaquarks, Phys. World 17, 25 (2004).

[2] B. Stech and Q.P. Xu, Z. Phys. C49 (1991) 491 and references therein.

[3] M. Cristoforetti, P. Faccioli, E. V. Shuryak and M. Traini, "Instantons, Diquarks and the Delta $(I)=$ 1/2 Rule for Non-Leptonic Hyperon Decays, Phys. Rev. D70, (2004) 054016.

[4] M. Cristoforetti, P. Faccioli, G.Ripka and M. Traini, Are there Diquarks in the Nucleon?, Phys. Rev. D 71, 114010 (2005)

[5] T. Schäfer and E.V. Shuryak, Instantons in QCD, Rev. Mod. Phys. 70 (1998) 323.

[6] P. Faccioli and E.V. Shuryak, A Systematic Study of the Single Instanton Approximation, Phys. Rev. D64, (2001) 114020. 